\title{
Minimally Invasive Aortic Arch Repair: Technical Considerations and Mid-Term Outcomes
}

\author{
Petar Risteski, ${ }^{1}$ Medhat Radwan, ${ }^{1}$ Gjoko Boshkoski, ${ }^{1}$ Razan Salem, ${ }^{1}$ Annarita Iavazzo, ${ }^{2}$ Thomas Walther, ${ }^{1}$ \\ Giampiero Esposito ${ }^{2}$ \\ ${ }^{1}$ Department of Thoracic and Cardiovascular Surgery, University Hospital Frankfurt, Frankfurt am Main, Germany; ${ }^{2}$ Mediterranea \\ Cardiocentro, Naples, Italy
}

\section{ABSTRACT}

Background: Reports of minimal invasive aortic arch surgery are scarce. We reviewed our experience with minimal access aortic arch surgery performed through an upper ministernotomy, with emphasis on details of operative technique and early and mid-term outcomes.

Methods: The medical records of 123 adult patients (mean age $66 \pm 12$ years), who underwent primary elective minimal access aortic arch surgery in two aortic referral centers, were reviewed. The most common indication was degenerative aortic arch aneurysm in 92 (75\%) patients. Standard operative and organ protection techniques used in all patients were upper mini-sternotomy, uninterrupted antegrade cerebral perfusion, and moderate systemic hypothermia $\left(27.4 \pm 1^{\circ} \mathrm{C}\right)$.

Results: Sixty-eight (55\%) patients received partial aortic arch replacement; the remaining $55(45 \%)$ patients received total arch replacement, further extended with either a frozen elephant trunk in $43(35 \%)$ patients or a conventional elephant trunk procedure in nine (7\%) patients. No conversion to full sternotomy was required. New permanent renal failure occurred in one $(0.8 \%)$ patient, stroke in two $(1.6 \%)$, and spinal cord injury in four (3.3\%) patients. Early mortality was observed in four (3.3\%) patients. At five years, survival was 80 $\pm 6 \%$ and freedom from reoperation was $96 \pm 3 \%$.

Conclusion: Minimal invasive aortic arch repair through an upper mini-sternotomy can be safely performed, with early and mid-term outcomes well comparable to series performed through a standard median sternotomy.

\section{INTRODUCTION}

Cardiac valve surgery through a less invasive incision has gained popularity over the last two decades. It has been shown to reduce bleeding, need for transfusion, operative time, ventilation time, and costs when compared with standard median sternotomy approach [Cohn 1997; Liu 1999; Mächler 1999].

Received May 26, 2020; accepted 7une 25, 2020.

Correspondence: Petar Risteski, MD, FETCS, Department of Thoracic and Cardiovascular Surgery, University Hospital Frankfurt, Theodor Stern Kai 7, Frankfurt am Main 60590, Germany; +4969-6301-5850; fax +4969-63015849 (e-mail: petarristeski@me.com).
Recent reports of surgery on the ascending aorta through a less invasive incision have proven the safety and feasibility of this approach [Byrne 2000; Svensson 2002; Tabata 2007]. Most reports consist of patients receiving replacement of the proximal aorta (ascending and root), whereas the inclusion of aortic arch procedures is inconsistent, varying from $5 \%$ [Tabata 2007] to $28 \%$ [Deschka 2013]. Thus, the analysis of outcomes after minimally invasive aortic arch repair (MIAAR) is quite difficult.

Literature on MIAAR is scarce [El-Sayed 2016; Goebel 2017; Risteski 2017]. Hereby, we report our two-center experience with MIAAR performed through an upper ministernotomy, with focus on operative technique details and early and mid-term outcomes.

\section{MATERIALS AND METHODS}

We reviewed the medical records of 123 adult patients (mean age $66 \pm 12$ years) who underwent primary elective aortic arch operation through an upper mini-sternotomy in two aortic referral centers between 2007-2019. The most common indication was degenerative aortic arch aneurysm in $92(75 \%)$ patients. Standard operative and organ protection techniques were used in all patients and included an upper mini-sternotomy, uninterrupted antegrade cerebral perfusion, and moderate systemic hypothermia $\left(27.4 \pm 1^{\circ} \mathrm{C}\right)$. The study was approved by the local ethics committee and individual consent was waived; the clinical data management was under the declaration of Helsinki.

Patient population: There were $68(55.3 \%)$ males. The most common indication was degenerative aortic arch aneurysm in $92(74.8 \%)$ patients, followed by chronic type A aortic dissection in $21(17.1 \%)$ patients. Patients' comorbidities included systemic arterial hypertension in 104 (84.6\%), chronic obstructive pulmonary disease in $20(17.3 \%)$, chronic renal insufficiency in 15 (12.2\%), and others (Table 1). Most common cardiac comorbidity was aortic valve insufficiency in $73(59.3 \%)$ patients. Patients with acute aortic dissection or elective cases with coronary artery disease requiring bypass grafting as well as patients coming for reoperative aortic arch procedure were operated through a full median sternotomy throughout the study period.

Operative and perfusion technique: The skin incision was started two finger breads below the jugular notch and extended caudally for 8-10 cm. The upper mini-sternotomy was " $\mathrm{L}$ "-ed 


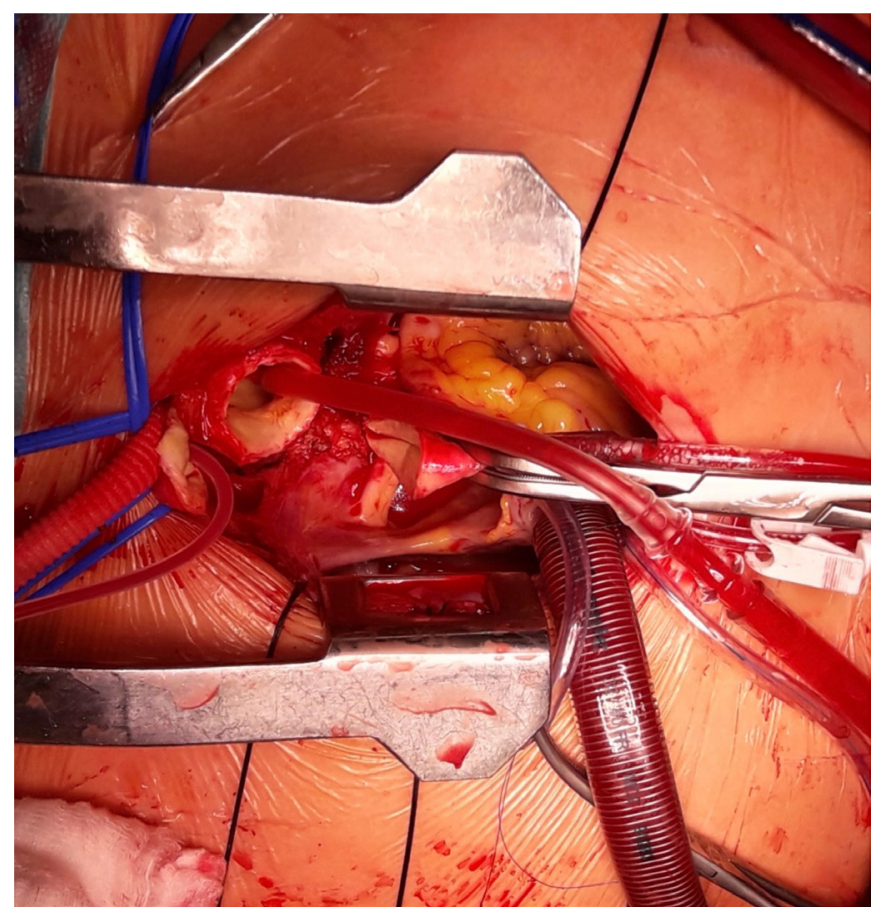

Figure 1. Intraoperative set up of minimal access aortic arch repair though an upper mini-sternotomy. Cranially, blue vessel loops are used to encircle the supraaortic branches. A $12 \mathrm{~F}$ soft tip cannula is inserted in the left common carotid artery for bilateral antegrade cerebral perfusion. The left subclavian artery has been debranched with an $8 \mathrm{~mm}$ polyester graft prosthesis, cannulated with a $18 \mathrm{~F}$ flexible cannula (not shown), and perfused. The aortic arch has been resected down to zone 2 , and a sucker is placed in the descending aorta. Caudally, the proximal ascending aorta has been clamped. A needle vent placed proximal to the clamp is used to decompress the left ventricle. A flattened doublestage venous cannula is used for drainage.

in the left fourth intercostal space and primary hemostasis was secured. Following systemic administration of heparin (400 IE $/ \mathrm{kg})$, the right axillary artery was exposed in the deltopectoral groove and directly cannulated using an 18-20 F flexible arterial cannula (EOPA Arterial Cannula, Medtronic, Dublin, Ireland). Pericardial incision was performed, and four stay sutures were placed. The three right-sided stay sutures were pulled upward, and the pericardium was delivered above the sternal edge. The fourth stay suture was placed at the left cranial aspect of the pericardiotomy incision and pulled caudally and upward. The right atrium was cannulated with a $28 \mathrm{~F}$ double-stage venous cannula (Edwards Lifesciences, Irvine, CA), the cardiopulmonary bypass was established using vacuumassisted drainage at $20-40 \mathrm{mmHg}$, and cooling was started. Insufflation of $\mathrm{CO}_{2}$ at a rate of 3 liters/minute was established. The left brachiocephalic vein was then mobilized, dissected free from its surroundings, and the supraaortic branches were exposed and encircled with elastomer loops. If required, debranching of the left subclavian artery was performed using an $8 \mathrm{~mm}$ polyester graft. The left ventricle was vented through the dome of the left atrium, and cold blood cardioplegia was administrated in antegrade fashion. Following cardioplegic
Table 1. Demographics of the study patients

$\begin{array}{lc}\text { N } & 123 \\ \text { Degenerative aneurysm (n, \%) } & 92(74.8) \\ \text { Chronic type A aortic dissection (n, \%) } & 21(17.1) \\ \text { Pseudoaneurysm (n, \%) } & 6(4.9) \\ \text { Porcelain aorta (n, \%) } & 4(3.3) \\ \text { Marfan syndrome (n, \%) } & 2(1.6) \\ \text { Arterial hypertension (n, \%) } & 104(84.6) \\ \text { Chronic obstructive pulmonary disease (n, \%) } & 20(17.3) \\ \text { Chronic renal insufficiency (n, \%) - on permanent dialysis (n, \%) } & 12.2) 0(0) \\ \text { Chronic peripheral arterial disease (n, \%) } & 5(4.1) \\ \text { Extracranial carotid disease (n, \%) } & 1(0.8) \\ \text { Preoperative neurologic dysfunction (n, \%) } & 2(1.6) \\ \text { Aortic valve stenosis (n, \%) } & 2(1.6) \\ \text { Aortic valve insufficiency (n, \%) } & 73(59.3) \\ \text { Aortic valve stenosis with insufficiency (n, \%) } & 17(13.8)\end{array}$

arrest and resection of the ascending aorta, the work on the aortic root was performed until the target temperature was reached. We aimed at $28^{\circ} \mathrm{C}$ body temperature, measured with a bladder and nasopharyngeal probes. Once the target temperature was reached, the supraaortic branches were occluded with the elastomers, the cross clamp was removed, and the antegrade cerebral perfusion was initiated. We used near infrared spectroscopy monitoring over the frontal cerebral cortex in addition to radial pressure monitoring to control the cerebral perfusion. After opening the aortic arch, the left subclavian artery, if intact, was blocked with a 7F Fogarty catheter, if snaring was not possible in cases with lateral displacement of the artery. Selective cannulation of the left common carotid artery was performed for bilateral antegrade cerebral perfusion using a balloon-tipped flexible arterial cannula if the ostium of the carotid artery was not severely altered with plaques, calcifications, or ulceration. The ACP was conducted with a perfusate temperature of $28-30^{\circ} \mathrm{C}$ with perfusion pressure kept at values greater than $75 \mathrm{~mm} \mathrm{Hg}$ as measured at the pump. At this point, the arch resection and repair was performed (Figure 1).

In patients with aneurysms extending into the proximal descending aorta, conventional or frozen elephant trunk with a hybrid prosthesis (Evita Open Plus, CryoLife JOTEC GmbH, Hechingen, Germany) or its "Light Arch" modification was employed [Gorlitzer 2007]. In Frankfurt, after completion of the arch repair and deairing, the graft was clamped, followed by reconstitution of full body perfusion [El-Sayed 2016]. In Bari, the lower body perfusion was established after deployment of the frozen elephant trunk prosthesis [Cappabianca 2017]. Proximal aortic repair followed during the rewarming period. In patients with staged treatment of an extensive aortic pathology (Figure 2), the second stage treatment was performed as soon as the kidney function returned to normal and the patient resumed his activities of daily life 
Table 2. Operative data

\begin{tabular}{lc}
\hline $\mathrm{N}$ & 123 \\
Operative time (min) & $346 \pm 66$ \\
Cardiopulmonary bypass time (min) & $178 \pm 44$ \\
Aortic cross-clamp time (min) & $116 \pm 36$ \\
Selective ACP (min) & $39 \pm 12$ \\
Distal core circulatory arrest time (min) in 98 patients in FFM & $39 \pm 12$ \\
Distal core circulatory arrest (min) in 25 patients in Bari & $15 \pm 4$ \\
Bilateral ACP (n, \%) & $72(58.6)$ \\
Lowest core temperature ( ${ }^{\circ}$ C) & $27.4 \pm 1$ \\
ACP flow $(\mathrm{l} /$ min) & $1.1 \pm 0.2$ \\
Hemiarch replacement (n, \%) & $68(55.3)$ \\
Total arch replacement (n, \%) & $55(44.7)$ \\
Classic & $3(2.4)$ \\
With conventional elephant trunk & $9(7)$ \\
With frozen elephant trunk & $43(35)$ \\
Aortic valve repair $/$ replacement (n, \%) & $41(33.3)$ \\
Valve sparing root replacement (David procedure) & $31(25.2)$ \\
Composite valve graft (Bentall procedure) & $9(7)$ \\
&
\end{tabular}

with full mobilization (usually 10-14 days following the first stage of treatment).

Data analysis: All preoperative, intraoperative, and postoperative data were analyzed using a central database. In order to evaluate the safety and effectiveness of this organ protection strategy, we evaluated the course of GOT (glutamicoxaloacetic transaminase), GPT (glutamic-pyruvic transaminase), $\gamma$-GT, serum lactate, serum creatinine, and blood urea nitrogen (BUN) at the following time points: preoperative and at 12 and 24 hours postoperatively. Furthermore, the acid-base balance was investigated in all patients by analysis of the serum lactate, the bicarbonate, base excess, and $\mathrm{pH}$. The definitions of permanent neurologic deficit (PND), temporary neurologic dysfunction (TND), and early mortality previously have been published [Zierer 2005]. The numerical rating scale (NRS) for pain and the administration of analgesics (Metamizol-Natrium-Monohydrat, Paracetamol and Piritramid) on demand during the hospital stay were used to assess the pain perception in this patient population.

Survivors were followed systematically through our outpatient clinic, and patients were advised to have an annual computed tomography scan. Study endpoints were morbidity and mortality, including mid-term survival and freedom from reoperation, which were plotted using the Kaplan-Meier analysis with the SPSS for Windows software (IBM, Armonk, USA).

\section{RESULTS}

Early results: In addition to replacement of the ascending aorta in all patients, hemiarch replacement was performed
Table 3. Early outcomes

\begin{tabular}{lc}
\hline $\mathrm{N}$ & 123 \\
Acute renal failure requiring dialysis $(\mathrm{n}, \%)$ & $12(9.8)$ \\
Temporary & $11(8.9)$ \\
Permanent & $1(0.8)$ \\
New malperfusion syndrome $(\mathrm{n}, \%)$ & $3(2.4)$ \\
New temporary neurologic deficit (n, \%) & $10(8.1)$ \\
New permanent neurologic deficit $(\mathrm{n}, \%)$ & $6(4.9)$ \\
Hemiplegia & $2(1.6)$ \\
Incomplete & $4(3.3)$ \\
Recurrent laryngeal nerve palsy (n, \%) & $2(1.6)$ \\
Ventilation time (h) & $8.5 \pm 3.3$ \\
Bleeding in 24 hour postoperatively (ml) & $390 \pm 250$ \\
ICU stay (days) & $1.6 \pm 0.6$ \\
Hospital stay (days) & $7 \pm 2$
\end{tabular}

in $68(55.3 \%)$ patients; the remaining 55 (44.7\%) patients received total arch replacement, with distal extension with either conventional in nine (7\%) patients or frozen elephant trunk in $43(35 \%)$ patients with extensive aortic pathology involving the proximal descending aorta. The average aortic cross clamp time was $116 \pm 36$ minutes and selective antegrade cerebral perfusion time was $39 \pm 12$ minutes, which was equal to visceral ischemia time in the population of patients operated in Frankfurt am Main. In Bari, distal body perfusion was established after deployment of the hybrid prosthesis, therefore, the visceral ischemia time was shorter with average of 15 \pm 4 minutes. In addition, $40(32.5 \%)$ patients received aortic root replacement, either valve-sparing root replacement in 31 $(25.2 \%)$ patients or Bentall procedure in nine $(7.3 \%)$ patients (Table 2).

There were no conversions to full sternotomy or other thoracic wall incisions. Three patients underwent second pump run, due to temporary right ventricular distention requiring additional reperfusion in one and residual bleeding in two, in the region of the aortic root after Bentall procedure in one and bleeding of a ligated left atrial appendage in another patient.

Early mortality was observed in four (3.3\%) patients. Neurologic morbidity included new temporary neurologic deficit in $10(8.1 \%)$ patients and new permanent neurologic deficit in six $(4.9 \%)$ patients (Table 3$)$.

Renal failure was present in 12 (9.8\%) patients, temporary in $11(8.9 \%)$ patients, and permanent in one $(0.8 \%)$ patient. Mean serum creatinine level for the study population rose from $0.96 \pm 0.31 \mathrm{mg} / \mathrm{dL}$ preoperatively to $1.04 \pm 0.42 \mathrm{mg} /$ $\mathrm{dL}$ at 12 hours postoperatively and to $1.73 \pm 0.4 \mathrm{mg} / \mathrm{dL}$ at 24 hours postoperatively, only to return to normal thereafter. Mean blood urea nitrogen increased from $38 \pm 14 \mathrm{mg} / \mathrm{dL}$ preoperative to $44 \pm 14 \mathrm{mg} / \mathrm{dL}$ at 12 hours and to $70 \pm 15 \mathrm{mg} / \mathrm{dL}$ at 24 hours after the surgery and normalized thereafter. Liver failure was not observed in the entire series: the mean preoperative GOT level from $38 \pm 10 \mathrm{mg} / \mathrm{dL}$ to $78 \pm 28 \mathrm{mg} / \mathrm{dL}$ 

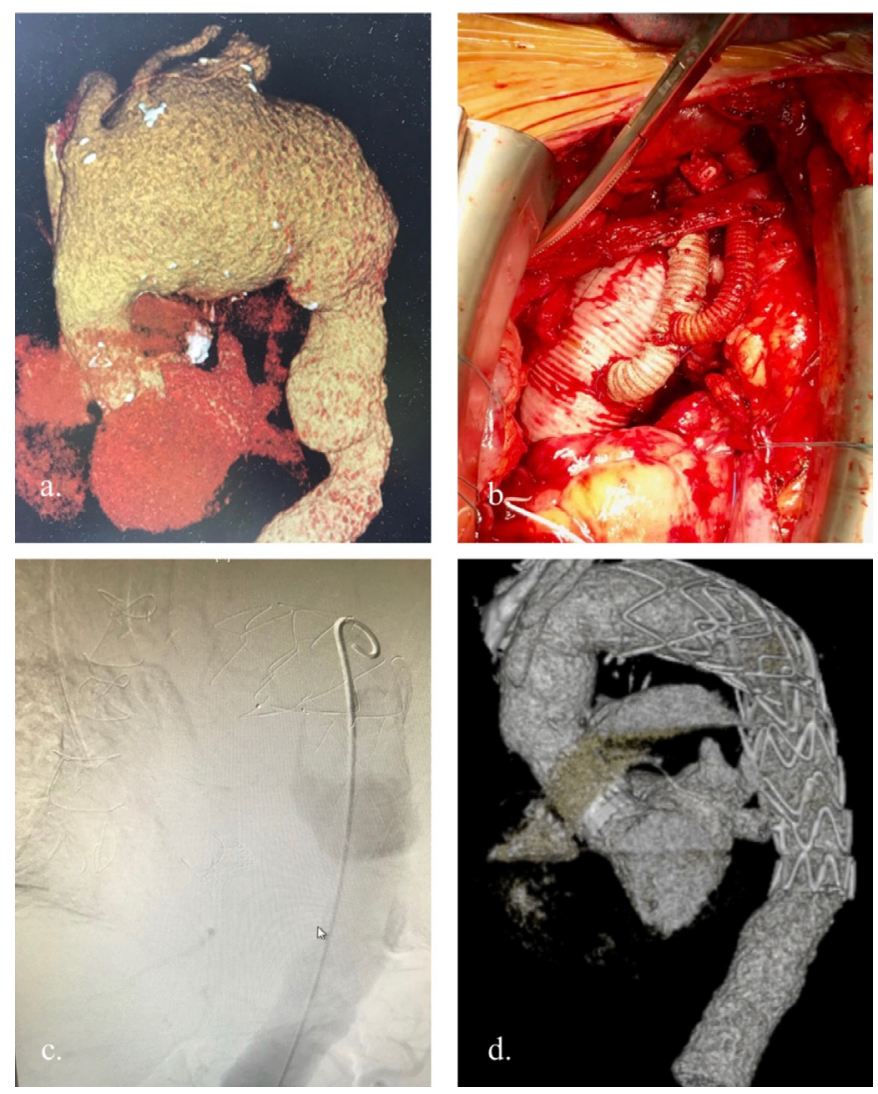

Figure 2. A) Two-stage treatment of a mega aorta syndrome, aortic valve stenosis and penetrating aortic ulcer of the descending aorta in a 78-year-old patient. B) Stage I treatment addressed the proximal aortic pathology through an upper mini-sternotomy with replacement of the ascending aorta, the aortic valve with a rapid deployment bioprosthesis, and replacement of the aortic arch with the frozen elephant trunk procedure, as well as debranching of the left common carotid artery and the left subclavian artery in zone 0. C) An anticipated Endoleak type $\mathrm{Ib}$ was treated on the tenth postoperative day during the second stage with a TEVAR extension (D).

at 12 hours postoperatively and finally to $128 \pm 34 \mathrm{mg} / \mathrm{dL}$ at 24 hours postoperatively, before returning to baseline values. Similar course was observed with the mean GPT value, which rose from $28 \pm 12 \mathrm{mg} / \mathrm{dL}$ preoperatively, to $62 \pm 22 \mathrm{mg} / \mathrm{dL}$ and to $96 \pm 25 \mathrm{mg} / \mathrm{dL}$ at 24 hours postoperatively, before returning to baseline mean. The mean values of $\gamma$-GT, serum lactate, serum bicarbonate, base deficit and $\mathrm{pH}$ displayed a similar course, during the early postoperative stay. Transfusion requirements, during the entire hospital stay, amounted to a mean of 1.66 red blood cells units per patient. Reopening for bleeding or tamponade was required in five (4\%) patients and three $(2.4 \%)$ patients, respectively, and was performed through the same incision. We did not observe any deep sternal wound dehiscence in this patient population.

Pain perception was assessed during the postoperative course (activities of daily living) of the patients. The mean NRS score was 2.49 at the first postoperative day and rose to 2.71 , during the second postoperative day, only to display a

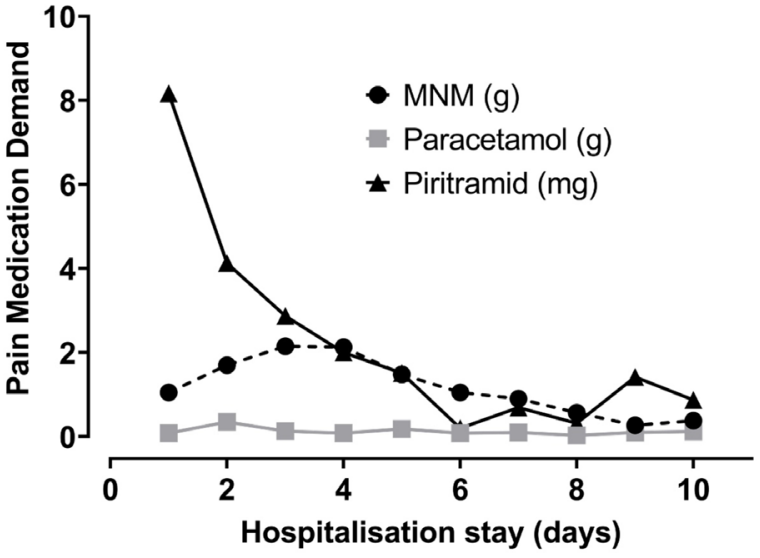

Figure 3. Demand of pain medications during the hospital stay.

descending course up to the fifth postoperative day (1.63) and remain at this level up to discharge day (1.58). Figure 3 shows the course of postoperative pain medication demand in the observed patient population (Figure 3 ).

Mid-term outcomes: One hundred and nineteen survivors were followed for 329 patient-years. Mean follow up was 33.2 \pm 16.2 months (range: $3-99$ ) and complete. Five patients died during follow up, due to intracranial bleeding, trauma, cancer, sepsis, and sudden cardiac death. Actuarial survival was $92 \pm 6$ $\%$ at three years and $80 \pm 11 \%$ at five years (Figure 4 ).

\section{DIscussion}

This observation underlines that minimally invasive aortic arch repair through an upper mini-sternotomy can safely be performed, with early and mid-term outcomes well comparable to series performed through a standard median sternotomy [Leshnower 2010; Zierer 2012]. The less invasive incision does not adversely influence the extent of resection of the aorta and the durability of aortic arch repair at mid-term.

Several minimal access approaches have been well established over the last two decades in cardiac valve surgery, like the upper or lower mini-sternotomy or right anterior minithoracotomy. The upper mini-sternotomy is our preferred approach for procedures on the aortic arch simply because the ascending aorta and aortic arch are located directly behind the upper sternum. Strategic placement of several pericardial stay sutures allow for the pericardium to be pulled upward and delivered above the sternum - a maneuver that brings the proximal aorta toward the surgeon. The size of the aneurysm was not found to be a limiting factor in successful execution of the operation thought a minimal access incision, as the larger the aneurysm is - more free space will be available for work after its resection. This is particularly convenient for patients with predominant pathology of the ascending aorta. For patients with predominant pathology in the region of the distal aortic arch and the proximal descending aorta, use of 


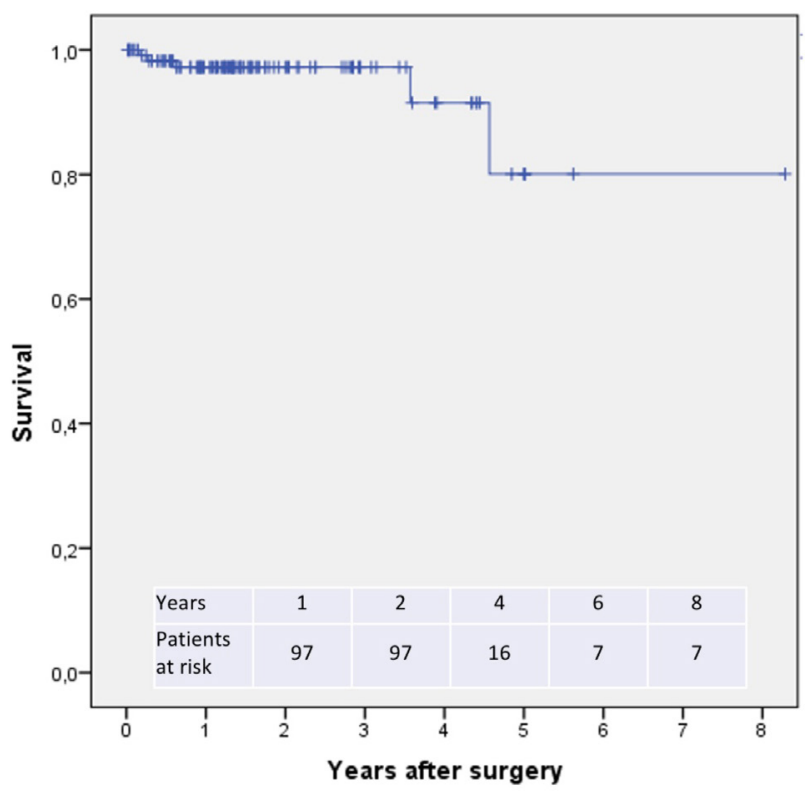

Figure 4. Mid-term survival

hybrid prosthesis has been the treatment of choice during the study period. The hybrid prosthesis allows the surgeon to limit the resection of the aneurysm to zone 1 (Figure 2) or zone 2 (Figure 1), and use the stent portion of the hybrid prosthesis to exclude the distal part of the pathology. Using this strategy, the surgeon does not have to work more distally, and, in our hands, zone 3 anastomosis was always avoidable.

Debranching of the left subclavian artery may indeed be the most difficult part of the operation. Several important technical aspects in performing debranching of the left subclavian artery include a full mobilization of the left carotid artery on cardiopulmonary bypass, proximal extension (usually $2-3 \mathrm{~cm}$ ) of the incision along the left sternocleidomastoid muscle, and partial division of the sternohyoid muscle. Using this strategy, we were able to reach and debranch the left subclavian artery in all instances except in six patients. If the left subclavian artery is displaced leftwards and not reachable though the upper mini-sternotomy, as assumed from the preoperative computed tomography imaging, two other options remain. A carotid-subclavian transposition before starting the aortic arch procedure allows for a zone 2 anastomosis; this was performed in two patients in our series. If the diameters of the carotid and subclavian arteries do not allow for a transposition or in case of other contraindication like extensive calcification, an extraanatomic bypass from the ascending aorta to the left axillary artery remains an option, and the surgeon can close the origin of the left subclavian artery through the open aortic arch and still do the distal anastomosis in zone 2. The latter approach was required in three patients in our series. If all these options fail, one still can choose to debranch the left subclavian artery at the time of the open arch repair at the cost of prolonging the antegrade cerebral perfusion (and visceral ischemia) time. This last option was required in only one patient in our series. Using these techniques, we never had to convert to full sternotomy or any other thoracic wall incision. The reasons for second pump run were such that can be also seen with full median sternotomy.

All surgeons performing the minimal access aortic arch repair in both institutions have had prior extensive experience with this incision in aortic valve, aortic root, and ascending aortic procedures. The standard-of-care organ protection protocol for aortic arch surgery as seen with the full median sternotomy does not have to be modified or altered with this limited incision. Indeed, the observed neurologic morbidity in these series is comparable with other reports on aortic surgery through minimal access [El-Sayed 2016; Svensson 2002; Svensson 2001; Shrestha 2015] or conventional approach [Leshnower 2010; Zierer 2012]. Additional analysis of the hepatic, renal, and visceral dysfunction sustained during the period of visceral ischemia demonstrates that this injury is temporary, mild and closely correlates with the one seen with conventional approach [Zierer 2012].

The same cannulas and instruments are used in both instances, with the upper mini-sternotomy as with the full median sternotomy. The left sided "L"-ing of the ministernotomy has been preferred, due to the fact that the aortic arch curves on to the left side, and in case of right-sided ascending aorta - the pericardial stay sutures will bring the ascending aorta in the middle of the incision. In extreme situations, one even can reach the posterior mediastinum and proximal descending aorta, even without a cardiopulmonary bypass [Risteski 2019].

Recently, Dr. Lamelas and coworkers reported their outcomes with the use of sternal-sparing minimal access surgery on the proximal aorta through a right mini-thoracotomy [Lamelas 2018]. Their report proves the feasibility and safety of performing operations on the proximal aorta (aortic root, ascending aorta, and proximal hemiarch repair) through a minimal right-sided access, but fails when it comes to more extensive arch repair, like total arch repair or even more distal aortic arch repair with the frozen elephant trunk procedure. Using the upper mini-sternotomy, we did not experience any relevant constrains in terms of exposition, did not have any conversion to full sternotomy, and did not observe any wound dehiscence in this study population. The pain observed with this limited incision has been only mild throughout and the need for analgesics consistently have been low throughout this series. Others have come to the same observations [Walther 1999] after minimally invasive heart valve operations.

The main limitation of this study is related to its retrospective nature. However, the incidence of serious complications is low, and therefore, identification of independent risk factors as well as the justification of this concept to grant even more patients access to this less invasive treatment remains to be evaluated by future research on larger cohorts.

\section{REFERENCES}

Byrne JG, Karavas AN, Cohn LH, Adams DH. 2000. Minimal access aortic root, valve and complex ascending aortic surgery. Current 
Cardiology Reports 2:549-557.

Cappabianca G, Roscitano C, Bichi S, et al. 2017. Whole body perfusion in patients undergoing frozen elephant trunk for type A acute aortic dissection. Perfusion 32:164-167.

Cohn L, Adams D, Couper G, et al. 1997. Minimally invasive cardiac valve surgery improves patient satisfaction while reducing costs of cardiac valve replacement and repair. Ann Surg 226:421-8.

Deschka H, Erler S, Machner M, et al. 2013. Surgery of the ascending aorta, root remodeling and aortic arch surgery with circulatory arrest through partial upper sternotomy: results of 50 consecutive cases. Eur J Cardiothoracic Surg 43:580-4.

El-Sayed Ahmad A, Risteski P, Papadopoulos N, et al. 2016. Minimally invasive approach for aortic arch surgery employing the frozen elephant trunk technique. Eur J Cardiothorac Surg 50(1):140-4.

Goebel N, Bonte D, Salehi-Gilani S, et al. 2017. Minimally invasive access aortic arch surgery. Innovations (Phila). 12(5):351-355.

Gorlitzer M, Weiss G, Thalmann M, et al. 2007. Combined surgical and endovascular repair of complex aortic pathologies with a new hybrid prosthesis. 84:1971-6.

Lamelas J, Chen P, Loor G, LaPietra A. 2018. Successful use of sternalsparing minimally invasive surgery of proximal ascending aortic pathology. Ann Thorac Surg 106:742-9.

Leshnower BG, Myung RJ, Kilgo PD, et al. 2010. Moderate hypothermia and unilateral selective antegrade cerebral perfusion: a contemporary cerebral protection strategy for aortic arch surgery. Ann Thorac Surg 90:547-554.

Liu J, Sidtropoulos A, Konertz W. 1999. Minimally invasive aortic valve replacement (AVR) compared to standard AVR. Eur J Cardiothoracic Surg 16(suppl 2):S80-3.
Mächler H, Bergmann P, Anelli-Monti M, et al. 1999. Minimally invasive versus conventional aortic valve operations: a prospective study in 120 patients. Ann Thorac Surg 67:1001-5.

Risteski P, El-Sayed Ahmad A, Monsefi N, et al. 2017. Minimally invasive aortic arch surgery; early and late outcomes. Int J Surg 45:113-7.

Risteski P, Radwan M, Mitrev Z, Walther T. 2019. Minimal access transposition of arteria lusoria through an upper mini-sternotomy. Clin Surg 4:2681-2.

Svensson LG. 2002. Progress in ascending and aortic arch surgery: minimally invasive surgery, blood conservation, and neurologic deficit prevention. Ann Thorac Surg 74:S1786-8.

Svensson LG, Nadolny EM, Kimmel WA. 2001. Minimal access aortic surgery including re-operations. Eur J Cardiothorac Surg 19:30-33.

Shrestha M, Krueger H, Umminger J, et al. 2015. Minimally invasive valve sparing aortic root replacement (David procedure) is safe. Ann Cardiothorac Surg 4:148-153.

Tabata M, Khalpey Z, Aranki SF, et al. 2007. Minimally access surgery of ascending and proximal arch of the aorta: a 9-Year experience. Ann Thorac Surg 84:67-72.

Walther T, Falk V, Metz S, et al. 1999. Pain and quality of life after minimally invasive versus conventional cardiac surgery. Ann Thorac Surg 67:1643-7.

Zierer A, Aybek T, Risteski P, et al. 2005. Moderate hypothermia $\left(30^{\circ} \mathrm{C}\right)$ for surgery of acute type A aortic dissections. Thorac Cardiovasc Surg $53: 74-9$.

Zierer A, El-Sayed Ahmad A, Papadopoulos N, et al. 2012. Selective antegrade cerebral perfusion and mild $\left(28-30^{\circ} \mathrm{C}\right)$ systemic hypothermic circulatory arrest for aortic arch replacement: results from 1002 patients. J Thorac Cardiovasc Surg 144:1042-1049. 